\title{
Diagnostic accuracy of C-reactive protein and white blood cell counts in the early detection of infectious complications after colorectal surgery
}

\author{
Pablo Ortega-Deballon • Olivier Facy • Patrick Rat
}

Accepted: 23 November 2011 / Published online: 9 December 2011

(C) Springer-Verlag 2011

\section{Dear Editor:}

We have read with great interest the article published by Warschkow et al. in the November issue of the Journal about the diagnostic accuracy of C-reactive protein (CRP) and white blood cells count (WBC) in the early detection of infectious complications after surgery for colorectal cancer. After a retrospective analysis of 1,187 patients who were operated on in their department over a 13-year period, they found that the highest diagnostic accuracy for the detection of postoperative inflammatory complications was observed for the CRP measured on postoperative day 4 (POD 4), with an area under the ROC curve (AUC) of 0.76 . The statistically optimal cut-off value at this time was $123 \mathrm{mg} / \mathrm{l}$, achieving a sensitivity of 0.66 . The accuracy obtained with the WBC counts was clearly lower.

The authors state that "there is no other study that directly compares the diagnostic impact of CRP and WBC in detecting inflammatory complications after colorectal surgery". They discuss several recent prospective studies which have shown the usefulness of CRP after colorectal surgery with criticism regarding their methods (inclusion criteria, inadequate analysis, absence of ROC analysis, not assessing CRP on POD 4).

In a recent prospective study published last year in the World Journal of Surgery, we included 133 patients undergoing elective colorectal surgery and compared CRP and WBC (with the "statistically adequate ROC analysis") for the detection of overall septic complications and anastomotic leakage. CRP was more accurate than the leukocyte count for

P. Ortega-Deballon $(\bowtie) \cdot$ O. Facy $\cdot$ P. Rat

Department of Digestive Surgical Oncology,

University Hospital of Dijon,

Dijon, France

e-mail: pablo.ortega-deballon@chu-dijon.fr

P. Ortega-Deballon $\cdot$ O. Facy $\cdot$ P. Rat

INSERM Unit 866 "Locoregional Therapy in Surgical Oncology",

Dijon, France

the detection of leakage or other septic complications in the postoperative setting. In particular, CRP on POD 4 yielded an AUC of 0.804 for the detection of postoperative septic complications. A cut-off value of $125 \mathrm{mg} / \mathrm{l}$ in the level of CRP at POD 4 yielded a sensitivity of $81.8 \%$ and a negative predictive value of $95.8 \%$ for the detection of anastomotic leakage.

Korner et al. communicated in the Journal of Gastrointestinal Surgery similar results with very close methods. They chose a higher cut-off rate (at POD 3) than ours at POD 4 (190 vs. $125 \mathrm{mg} / \mathrm{l})$, obtaining a similar sensitivity (82\%) for the detection of postoperative complications. The peak of postoperative CRP happens between POD 2 and 3 and then the values decrease. This may explain why the cut-off value obtained at POD 3 by Korner et al. was higher than ours, which was obtained at POD 4. This tallying between the three works is a strong argument in favour of the usefulness of the CRP in this setting. As we concluded in our article, "patients with CRP values lower than $125 \mathrm{mg} / \mathrm{l}$ on POD 4 can be safely discharged from hospital". An ongoing French prospective multicentric trial is now comparing the accuracy of CRP and procalcitonin after colorectal surgery. In the context of this study, patients with CRP values higher than $125 \mathrm{mg} / \mathrm{l}$ at POD 4 systematically undergo an abdominal CT scan to search for a surgical site infection.

Finally, the authors had an unusually long overall median hospital stay of $20.7 \pm 12.2$ days due to the current hospital policy in Switzerland. They find that "the long hospital stay is an advantage because the detection rate for complications may be higher". However, no data are given in the study regarding the timing of onset or discovery of postoperative complications. The laboratory data are given only until POD 5. There are no arguments in their article to support the benefit of such a long hospital stay. At any rate, clinically significant complications are detected out of the hospital and usually induce readmissions. Their long hospital stay cannot be considered as an advantage but could be attributed as a particularity of their health system. 